\title{
Status of onchocerciasis in Teppi area, Southwestern Ethiopia, after four years of annual community-directed treatment with ivermectin
}

\author{
Mengistu Legesse ${ }^{1}$, Fekede Balcha ${ }^{1}$, Berhanu Erko ${ }^{1}$
}

\begin{abstract}
Background: Information on the impact of annual community-directed treatment with ivermectin (CDTI) on the prevalence and intensity of onchocerciasis as well as its transmission following years of repeated ivermectin distribution is vital to monitor the impact of the control efforts.

Objective: To determine the status of the prevalence and intensity of onchocerciasis after four years of ivermectin distribution in Teppi area.

Methods: Skin snips were obtained from 390 study participants and kept in physiological saline at room temperature for 24 hours. The emerged microfilariae were counted under 10x microscope objective. The skin snips were weighed and the microfilarial load was expressed as the arithmetic mean of two skin snips per mg of skin snip.

Results: Out of the total 390 examined subjects, $87(22.3 \%)$ were found to be positive for microfilaridermia. Significant differences in the prevalence and microfilarial load of the disease were not found among untreated and treated subjects $(\mathrm{p}>0.05)$. Significant reductions of the prevalence $(25.5 \%)$ and intensity of infection $(0.08-8 \mathrm{mf}$, mean 1.3) were found compared to prevalence (81\%) and intensity of infection ( $0-855$, mean $33 \pm 5.6)$ reported before the initiation of the CDTI programme in the study area.

Conclusion: The overall results of the study showed a high reduction both in the prevalence and intensity of infection compared to prevalence and intensity of the disease before the initiation of the CDTI programme in the study area. Nevertheless, continued assessment of the long-term impact of repeated annual CDTI programme on onchocerciasis and identification of factors that could affect the sustainability of the programme in various CDTI areas of Ethiopia, could contribute towards the effective control of the programme. [Ethiop. J. Health Dev. 2010;24(1):51-56]
\end{abstract}

\section{Introduction}

Onchocerciasis is a disease of public health and socioeconomic concern in many African countries. The ultimate goal of the African Programme for Onchocerciasis Control (APOC) is to reduce the public health and socio-economic problems of onchocerciasis within a period of 12-15 years using the strategy of yearly community-directed treatment with ivermectin (CDTI) in endemic areas. Ivermectin is highly appreciated for its effectiveness in the mass treatment of onchocerciasis (1). However, it is important to assess the impact of annual CDTI on the prevalence/transmission of onchocerciasis and community microfilarial load (CMFL) following years of repeated annual ivermectin distribution in order to monitor the direction of the control efforts (2-4).

In Ethiopia, onchocerciasis has been known to be endemic in several localities of the Southwestern and Northwestern parts in different magnitudes of endemicity (5-8). A countrywide Rapid Epidemiological Mapping of Onchocerciasis (REMO) carried out in 1997 and 2001 by APOC in collaboration with the Ethiopian Federal Ministry of Health revealed that onchocerciasis was much more widespread in Ethiopia than previously estimated (9). Based on the REMO results of 1997, a community-based free distribution of ivermectin was first launched in Sheka Zone, Southwestern Ethiopia in the year 2001 and then in the rest of endemic regions phase by phase (10).

Prior to the initiation of CDTI programme in Teppi area, Mengistu et al. (11) studied the prevalence and intensity of onchocerciasis in residents of Endris-Goji and Addis Berhane villages. In this study, they reported a prevalence of $81 \%$ and a microfilarial load ranging from $0-855$ (mean $33 \pm 5.6$ ) in the age group 11-78 years. Although the CDTI programme is in its $4^{\text {th }}$ year of implementation period in Teppi area, there is no report on the impact of annual CDTI on the magnitude of onchocerciasis after the implementation of the programme in the area. Therefore, the aim of this study was to determine the status of the prevalence and intensity of onchocerciasis after four years of ivermectin distribution using the pre-intervention information on the prevalence and intensity of the disease in same study area.

\section{Methods}

\section{Study Area and Population}

In February 2005, a cross-sectional parasitological survey was conducted in three adjacent villages (Endris-Goji, Addis Berhane and Nupa) around Teppi town, to assess the prevalence and intensity of onchocerciasis after four years of ivermectin distribution. The villages are found $630 \mathrm{~km}$ to the Southwest of Addis Ababa and about $8 \mathrm{~km}$

Aklilu Lemma Institute of Pathobiology, Addis Ababa University, P. O. Box 1176, Fax: 00251-1-755296/550655, Email: dlegessem@yahoo.com, Addis Ababa,Ethiopia 
to the Southeast of Teppi town. The majority of inhabitants of Endris-Goji were the indigenous Mezhenger ethnic group, while the inhabitants of Addis Brehane and Nupa were a mixture of Amahara and Kafa ethnic group who came to the area from other parts of Ethiopia. Most of the inhabitants were engaged in mixed farming, coffee plantation, maize cultivation and rearing livestock. According to information obtained from the leader of the villages, there were about 673 households with a total of approximately 4365 population in the three villages. The inhabitants of the villages were living under similar setting. A more detailed description of the study area are given elsewhere (11).

In the study villages, community based ivermectin (Mectizan ${ }^{\circledR}$ ) treatment for onchocerciasis was initiated in October 2001. However, during the second round of the treatment it was delayed for five months because of the month of October is the harvest time in the area. The second round ivermectin distribution was performed in March 2002 and then annually distributed to all eligible members of the communities in the villages through community drug distributors using the height and physical appearance dosing method (12). This survey was conducted one month prior to the fifth round of annual ivermectin distribution in the area. According to the information obtained from community drug distributors and District Health Office, the annual treatment coverage of ivermectin was high (over 90\%) and sustainable in the study area. Adults and children (age over 5 years) who lived in the area for more than one year, ivermectin treated or untreated, but volunteered to participate were included in the study.

\section{Sample Size Estimation}

Pre-intervention information on the prevalence of onchocerciasis $(81 \%)$ in the study villages (11) was used as background information to estimate the sample size. However, since the CDTI programme was in its $4^{\text {th }}$ annual round in the study area during the survey, a $20 \%$ reduction in the prevalence of the disease due to the effect of the intervention was assumed (13). Hence, a total of 388 (after adding 5\% compensation for reluctant cases) study participants were considered to be included in the study.

\section{Sample Collection and Examination}

After explaining the aim of the study and obtaining informed consent from the leaders of the villages, the total number of households in the study villages was estimated. Then, house-to-house visit was made to inform randomly selected (random selection was done using the lists of households) head of the households to come to a central place with their eligible families (both male and female, older than 5 years) for onchocerciasis examination. Then, two skin snips, one from the left and the other from the right iliac crest were taken from each study participants under aseptic condition using disposable sterile pricking needle and razor blade. The skin snips were then separately placed in flat-bottomed micro-titer plate wells filled with $100 \mu 1$ of physiological saline and left at room temperature for 24 hours. About four drops of $4 \%$ formalin were added into each well and the samples were transported to laboratory as previously described $(7,8,11)$. The skin snip from each well was collected onto a filter paper and the whole content in individual well was transferred onto a microscopic slide. The emerged microfilariae were counted under $10 \mathrm{x}$ microscope objective. The skin snips from each subject were weighed using analytical balance (Mettler AE 260 Delta Range) after avoiding excess water by tapping on absorbent filter paper. The microfilarial load of each positive subject was calculated as the arithmetic mean of two skin snips per mg of skin snip $(8,11)$. The CMFL was calculated only for those study participants who were 20 years and older as previously recommended (14). Pre-intervention information on the prevalence and intensity of onchocerciasis (11) was used as background information to assess the impact of ongoing CDTI programme on the prevalence of the disease. Information on age, gender as well as history of treatment with ivermectin were collected during the survey.

\section{Data Analysis}

The percentage of individuals with onchocerciasis was determined by dividing the total number of positive individuals for $O$. volvulus infection by the total number of study participants. Chi-square $\left(\mathrm{X}^{2}\right)$ test was used to compare the proportion of infection among the different categories of the study subjects. Arithmetic mean was used to determine the intensity of infections. Student ttest was also used to compare the means of microfilarial load between the different study groups. Differences were considered to be significant when $p$ value less than 0.05 .

\section{Ethical Consideration}

The study was ethically approved by the Ethical Clearance Committee of Aklilu Lemma Institute of Pathobiology, Addis Ababa University. The objective of the study was explained to the participants or their guardians in local language. Skin snip samples were taken after informed verbal consent was obtained. Positive individuals for onchocerciasis were advised to receive ivermectin treatment during the scheduled distribution. Negative individuals were also advised to continue receiving the regular annual treatment.

\section{Results}

A total of 390 study participants (202 females and 188 males) in the age range of 5-85 years (mean 21.7) volunteered to provide skin snips. The sociodemographic characteristic of the study participants is shown in Table 1. Out of 390 examined subjects, 87 $(22.3 \%)$ were found positive for microfilaridermia. Significant differences in the prevalence of the disease were not found between males and females or among different age groups $(\mathrm{p}>0.05)$ (Table 2). However, the

Ethiop. J.Health Dev. 2010;24(1) 
prevalence of the disease was found to be significantly higher in the study participants from Endris-Goji village compared to study subjects from Addis Berhane $(\mathrm{p}<$ $0.001)$ and Nupa $(p<0.05)$ villages. The prevalence of the disease was also found to be significantly higher in the study participants who lived over 10 years in the area $(p<0.05)$. The overall microfilarial load of the positive individuals ranged from $0.08-8 \mathrm{mf}$ (mean 1.2) per gm of skin snip. Table 3 shows the status of treatment with ivermectin versus prevalence of onchocerciasis and microfilarial load of the study participants positive for microfilaridermia.

Table 1: Socio-demographic characteristics of the study participants, Teppi area, Southwestern Ethiopia, February 2005

\begin{tabular}{ll}
\hline \multicolumn{1}{c}{ Variable } & Number(\%) \\
\hline Gender of participants & $188(48.2)$ \\
Male & $202(51.8)$ \\
Female & \\
Age group (year) & $166(42.6)$ \\
$5-14$ & $74(19.0)$ \\
$15-24$ & $71(18.2)$ \\
$25-34$ & $79(20.2)$ \\
$35+$ & \\
Ethnic Group & $163(41.8)$ \\
Mesjenger & $126(32.3)$ \\
Amahra & $91(23.3)$ \\
Kafa & $10(2.6)$ \\
Other & \\
Village & $141(36.2)$ \\
Endris-Goji & $157(40.2)$ \\
Addis Berhane & $92(23.6)$ \\
Nupa & \\
Length of residence (year) & $102(26.2)$ \\
1-9 & $123(31.5)$ \\
10-19 & $165(42.3)$ \\
20+ &
\end{tabular}

Significant differences in the prevalence of onchocerciasis were not found among the percentages of the untreated, less than four round treated and four round treated groups $(\mathrm{p}>0.05)$. Similarly, the microfilarial load did not significantly differ among the groups. The overall CMFL for 192 study participants (including those with negative counts) who were 20 years and older ranged from $0-8 \mathrm{mf}$ (mean 0.38 ) per $\mathrm{mg}$ of skin snip. However, the CMFL for those 48 positive subjects (age over 20 years) ranged from $0.08-8 \mathrm{mf}$ (mean 1.5 ) per $\mathrm{mg}$ of skin snip.

Table 2: Prevalence of onchocerciasis according gender, age, village and length of residence, Teppi area, Southwestern Ethiopia, February 2005

\begin{tabular}{ll}
\hline Variable & $\begin{array}{l}\text { Number Positive } \\
(\%)\end{array}$ \\
\hline $\begin{array}{l}\text { Gender } \\
\text { Male }\end{array}$ & $46(24.5)$ \\
Female & $41(20.3)$ \\
Age group (year) & $31(18.7)$ \\
$5-14$ & $19(25.7)$ \\
$15-24$ & $18(25.4)$ \\
$25-34$ & $19(24.1)$ \\
$35+$ & $46(32.6)^{*}$ \\
Village & $23(14.6)^{*}$ \\
Endris-Goji & $18(19.6)^{*}$ \\
Addis Berhane & \\
Nupa & $14(13.7)^{*}$ \\
Length of residence (year) & $31(25.2)$ \\
1-9 & $42(25.5)^{*}$ \\
$10-19$ & \\
$20+$ &
\end{tabular}

Table 3: Prevalence of onchocerciasis and microfilarial load in all the study participants,

Teppi area, Southwestern Ethiopia, February 2005

\begin{tabular}{lllll}
$\begin{array}{l}\text { Treatment } \\
\text { status }\end{array}$ & $\begin{array}{l}\text { Number of } \\
\text { subjects }\end{array}$ & $\begin{array}{l}\text { Age range } \\
\text { (mean) }\end{array}$ & Number Positive (\%) & $\begin{array}{l}\text { MFL range (mean)/mg of } \\
\text { skin }\end{array}$ \\
\hline Untreated & 24 & $5-35(11.1)$ & $4(16.7 \%)$ & $0.25-1.7(0.83)$ \\
$1^{\text {st }}$ round & 44 & $6-70(12.7)$ & $13(29.5 \%)$ & $0.13-5.5(1.7)$ \\
$2^{\text {nd }}$ round & 76 & $7-55(14.1)$ & $16(21.1 \%)$ & $0.18-7.8(1.5)$ \\
$3^{\text {rd }}$ round & 68 & $8-85(20.3)$ & $15(22.1 \%)$ & $0.11-2.5(0.69)$ \\
$4^{\text {th }}$ round & 178 & $8-70(29.3)$ & $39(21.9 \%)$ & $0.08-8(1.2)$
\end{tabular}

Previous study conducted in the same villages before the initiation of the CDTI programme involved individuals in the age group 11-78 years. In order to compare the present prevalence and intensity of onchocerciasis with the baseline prevalence and intensity, individuals younger than 10 years were excluded, and data was analyzed for 271 (134 males and 137 females , age rang $10-85$, mean 28.0 years) participants. Of these, $69(25.5 \%)$ individuals were found positive for microfilaridermia. The overall microfilarial load of the positive individuals ranged from $0.08-8 \mathrm{mf}$ (mean 1.3) per gm of skin snip. The present prevalence $(25.5 \%)$ and intensity of infection are significantly lower than prevalence $(81 \%)$ and intensity of infection $(0-855$, mean $33 \pm 5.6)$ reported before the initiation of the CDTI programme in the study area. Table 4 shows the status of treatment with ivermectin versus prevalence of onchocerciasis and microfilarial load of the study participants whose age was over 10 years. 
Table 4: Prevalence of onchocerciasis and microfilarial load in study participants age 10 years and above, Teppi area, February 2005

\begin{tabular}{lllll}
$\begin{array}{l}\text { Treatment } \\
\text { status }\end{array}$ & $\begin{array}{l}\text { Number of } \\
\text { subjects }\end{array}$ & $\begin{array}{l}\text { Age range } \\
\text { (mean) }\end{array}$ & $\begin{array}{l}\text { Number Positive } \\
(\%)\end{array}$ & $\begin{array}{l}\text { MFL range (mean)/mg of } \\
\text { skin }\end{array}$ \\
\hline $\begin{array}{l}\text { Untreated } \\
1^{\text {st }} \text { round }\end{array}$ & 8 & $13-35(24.3)$ & $3(37.5 \%)$ & $0.4-1.7(1.0)$ \\
$2^{\text {nd }}$ round & 35 & $20-70(34.3)$ & $6(66.7 \%)$ & $0.25-5.5(2.1)$ \\
$3^{\text {rd }}$ round & 53 & $11-55(21.1)$ & $9(25.7 \%)$ & $0.18-7.8(1.8)$ \\
$4^{\text {th }}$ round & 166 & $11-85(23.7)$ & $11(20.8 \%)$ & $0.11-2.5(0.84)$ \\
\hline
\end{tabular}

\section{Discussion}

In this study pre-intervention information on the prevalence and intensity of onchocerciasis (11) was used as background information to assess the impact of ongoing CDTI programme on onchocerciasis in untreated and subjects at various rounds of treatment in an area where the fourth round of annual CDTI was completed. The overall results showed a marked reduction in both the prevalence $(22.3 \%)$ and the intensity of infection (0-8 $\mathrm{mf} / \mathrm{gm}$ of skin snip, mean 1.2) compared to the prevalence $(81 \%)$ and intensity $(0-855, \mathrm{mf} / \mathrm{gm}$ of skin snip, mean 33) of infection reported prior to the initiation of CDTI programme in the study area (11). One major difference between the present and the previous study was that the present study included individuals in the age range 5-85 years, while the previous one considered individuals' age ranging from 11- 78 years. Nevertheless, the observed high reduction rate in the prevalence and intensity of infection remained high even when individuals younger than 10 years were excluded from data analysis. The findings of the present study also corroborated the findings of others that repeated ivermectin treatments can result in a profound reduction in the transmission/prevalence of onchocerciasis as well as in the intensity of infection even in the areas where vector control activities have not been conducted $(2,15-$ 18).

Our study also showed that relatively a higher prevalence of onchocerciasis among study participants from EndrisGoji village than from the other villages, which is in agreement with the result of previous study conducted in the area (11). This might possibly be attributed to their life style that the Mezhengers are hunters, honey collectors, activities which increase exposure and reexposure to fly bites and could lead to super infection (19). It could also be explained by a longer duration of exposure to the disease by native inhabitants as evidenced in the present study.

Studies in Burundi (20) and Cameroon (13) have shown a high reduction in the prevalence and the intensity of microfilaridermia in young children who have never received ivermectin, but lived in four annual rounds of mass ivermectin treated communities. In agreement with these previous findings, we found absence of significant differences in the prevalence of onchocerciasis among the untreated and four round treated study participants. A significant reduction in microfilarial load was observed following four rounds of repeated annual treatments of onchocerciasis with ivermectin in six of the 10 communities studied in Nigeria (4). Contrary to this finding, the present study revealed absence of significant difference in microfilarial load among untreated, less than four round treated and four round treated study participants. Further study involving large sample size may be needed to investigate the discrepancy between the present and this previous study.

The present study indicated that the on-going mass ivermectin treatment has been effective in reducing the prevalence of onchocerciasis though its long-term impact and success in eradication of the disease needs to be followed. As parasitological assessment in human host alone cannot confirm the impact of mass ivermectin treatment on the transmission of onchocerciasis, the impact of CDTI on parasite in the blackfly vector need to be assessed even if method of data collection and interpretation of results are difficult as the transmission potentials may vary from year to year and the larvae of O.volvulus closely resemble those of O.ochengi, a parasite of cattle transmitted by blackflies of the same species $(21,22)$.

The success of the CDTI progrmme can be affected by factors such as the level of community awareness about the cause, mode of transmission and preventive methods of the disease, inadequate and poor community mobilization, village characteristics, system of drug distribution, conflict among different ethnic groups and the motivation of community drug distributors (CDDs) (23-27). Hence, assessment of community perception about the disease and the CDTI program, and timely report on community response to the programme could help to manage and sustain the annually ivermectin distribution activity in the study area.

In conclusion, the results of the present study showed a considerable reduction both in the prevalence and intensity of onchocerciasis in the study area, after four years of annual CDTI compared to the prevalence and intensity of infection of the disease prior to the initiation of CDTI programme. Since no other control measures have been implemented in the study area, the present 
observed reduction in prevalence as well as intensity of infection can be attributed to the consecutive ivermectin distribution and high treatment coverage. Nevertheless, continued assessment of the long-term impact of repeated annual CDTI programme on onchocerciasis and identification of factors that could affect the sustainability of the programme in various CDTI areas of Ethiopia, could contribute towards the effective control of the programme.

\section{Acknowledgments}

The authors would like to acknowledge financial support provided by the Aklilu Lemma Institute of Pathobiology, Addis Ababa University and the technical assistance rendered by technical staff of Medical Parasitology Unit. The community leaders in the study area and the participants are also duly acknowledged for their cooperation during the survey.

\section{References}

1. Tielsch JM, Beeche A. Impact of ivermectin on illness and disability associated with onchoceciasis. Trop Med Intern Health 2004;9:A45-A56.

2. Alley ES, Plaisier AP, Boatin BA, Dadzie KY, Remme J, Zerbo G, Samba EM. The impact of five years of annual ivermectin treatment on skin microfilarial loads in the onchocerciasis focus of Asubende, Ghana. Trans Roy Soc Trop Med Hyg 1994;88:581-584.

3. Dadzie Y, Neira M, Hopkins D. Final report of the Conference on the eradicability of onchocerciasis. Filaria J 2003; 2: 2.

4. Oyibo WA, Fagbenro-Beyioku AF. Effect of repeated community-based ivermectin treatment on the intensity of onchocerciasis in Nigeria. Rural Remote Health 2003;3:1-9.

5. Zein A Z. The epidemiology of onchocerciasis in northwestern Ethiopia. Trop Geograp Med 1986;38:33-37.

6. Gundersen S G, Schmitt-Lechner A, Bjorvatn B. Onchocerciasis in the Blue Nile Valley of western Ethiopia. Trans Roy Soc Trop Med Hyg 1988;82:122-127.

7. Taye A, Gebre-Michael T, Taticheff S. Onchocerciasis in Gilgel Ghibe River Valley southwestern Ethiopia. East Afr Med J 2000;77:116-120.

8. Hailu A, Balcha F, Birrie $H$, Berhe $N$, Aga A, Mengistu G, Bezuneh A, Ali A, Gebre-Michael T, Gemetchu T. Prevalence of onchocercal skin disease and infection among workers of coffee plantation farms in Teppi, southwestern Ethiopia. Ethiop Med J 2002;40:259-69.

9. Carter Center. Summary: 2001 program Review for the Carter Center/Lions SightFirst River Blindness Programs Cameroon, Ethiopia, Nigeria, OEPA, Sudan and Uganda 13-15 March, 2002. The Carter Center Atlanta, GA.
10. Taticheff S, Alamrew D, Gebre T. Onchocerciasis. In: Epidemiology and ecology of health and diseases in Ethiopia. 2006. p. 591-608.

11. Mengistu G, Balcha F, Britton S. Co-infection of Onchocerca volvulus and intestinal helminths in indigenous and migrant farmers in southwest Ethiopia. Ethiop Med J 2002;40:19-27.

12. Taylor HR, Gonzales C, Duke B. Simplified dose schedule of ivermectin. Lancet, 1993;341:50-51.

13. Pion S DS, Clement MCA, Boussinesq M. Impact of four years of large-scale ivermectin treatment with low therapeutic coverage on the transmission of Onchocerca volvulus in the Mbam Valley focus, central Cameroon. Trans Roy Soc Trop Med Hyg 2004;98:520-528.

14. Remme J, Ba O, Dadzie KY, Karam M. A forceof-infection model for onchocerciasis and its application in the epidemiological evaluation of the onchocerciasis control programme in the Volta River Basin area. Bulletin of the WHO 1986;64:667-81.

15. Taylor HR, Pacque M, Munoz B, Greene BM. Impact of mass treatment of onchocerciasis with ivermectin on the transmission of infection. Science 1990;250:116-8.

16. Boussinesq M, Chippaux JP, Ernould JC, Quillevere D, Prod'hon J. Effect of repeated treatments with ivermectin on the incidence of onchocerciasis in northern Cameroon. Am J Trop Med Hyg 1995;53:63-67.

17. Boatin BA, Hougard JM, Alley ES, Akpoboua LKB, Yameogo L, Dembele N, Seketeli A, Dadzie KY. The impact of Mectizan on the transmission of onchocerciasis. Annal Trop Med Pararsitol 1998;92:S47-S60.

18. Kennedy MH, Bertocchi I, Hopkins AD, Meredith SE. The effect of 5 years of annual treatment with ivermectin (Mectizane) on the prevalence and morbidity of onchocerciasis in the village of Gami in the Central African Republic. Annal Trop Med Parasitol 2002;96:297-307.

19. Meludu NT, Ajayi O O. Effect of onchcerciasis on farming activity in Oyo State, Nigeria. Afr J Biomed Res 2005;8:143-149.

20. Newell E D. Effect of mass treatments with ivermectin, with only partial compliance, on prevalence and intensity of O.volvulus infection in adults and in untreated 4 and 5 years-old children in Burundi. Trop Med Intern Health 1997;2:912-6.

21. Renz A. Studies on the dynamics of transmission of onchocerciasis in a Sudan-savanna area of North Cameroon II. Seasonal and diurnal changes in the biting densities and in the age-composition of the vector population. Ann Trop Med Parasitol 1987;81:229-37.

22. Wahl G, Ekale D, Schmitz A. Onchocerca ochengi: assessment of the Simulium vectors in north Cameroon. Parasitol 1998;116:327-336.

23. Hewlett BS, Kollo B, Cline BL. Ivermectin distribution and the cultural context of forest

Ethiop J Health Dev. 2010;24(1) 
onchocerciasis in South Province, Cameroon. Am J Trop Med Hyg 1996;54:517-22.

24. Gardon J, Mace J.M, Cadot E, Ogil C, Godin C, Boussinesq $\mathrm{M}$. Ivermectin-based control of onchocerciasis in northern Cameroon: individual factors influencing participation in community treatment. Trans Roy Soc Trop Med Hyg 1996;90:218-22.

25. Oyibo WA, Fagbenro-Beyiku AF. Evaluation of community compliance with annual ivermectin treatment of onchocerciasis in Patigi, Nigeria. East Afr Med J 1998;5:237-42.
26. Brieger WR, Otusanya SA, Oke GA, Oshiname FO, Adeniyi JD. Factors associated with coverage in community-directed treatment with ivermectin for onchocerciasis control in Oyo State, Nigeria. Trop Med Internat Health 2002;7:11-8.

27. Emukah EC, Enyinnaya U, Olaniran NS, et al. Factors affectiving the attrition of community directed distributors of ivermectin, in an onchocerciasis control programme in the Imo and Abia states of south-eastern Nigeria. Annal Trop Med Parasitol 2008;102:45-51. 